\title{
111 - Surface neuromodulation (TMS and tDCS) for therapy of cognitive and psychiatric disorders.
}

Coordinator Dr. Howard Chertkow, University of Toronto (Baycrest Health Sciences), Canada.

The field of neuromodulation has progressed significantly over the past two decades. It is evident that application of electrical (via tDCS, transcranial direct current stimulation) or magnetic (via rTMS, repetitive transcranial magnetic stimulation) brain stimulation over the skull surface can effect change in brain function, which appears sufficiently robust to have a therapeutic effect. Sometimes the neuromodulation is best coupled with other forms of training or rehabilitation for best efficacy. What are the most promising approaches? What conditions appear to benefit? What are the situations/diseases/ disease states where neuromodulation is sufficiently well-proven now (or may be so in the future) that clinicians should start to consider its use in their psychogeriatric practice? We will review studies showing that tDCS can have a therapeutic effect in dementia, stroke, depression, and a range of other psychiatric conditions. Recent work is showing that with tDCS one can achieve improvement in picture naming, executive function, and memory in Alzheimer Disease and Frontotemporal dementia (Howard Chertkow presentation, Baycrest Health Sciences, Toronto). In stroke rehabilitation, rTMS treatment has been shown to aid in motor and language recovery (Alex Thiel, McGill University). There is now sufficient evidence that tDCS and Magnetic Seizure therapy are beneficial in depression, that these can now become part of the therapeutic armamentarium in selected cases (Jeff Daskalakis, University of Toronto). A range of other neuropsychiatric conditions can also be considered for neuromodulation therapy with rTMS (Daniel Blumberger, University of Toronto, $\mathrm{CAMH}$ ).By attending this symposium, a physician or health care professional will become familiar with the latest research into neuromodulation and its role in current therapy of neurological and psychiatric diseases. 